\title{
CHANGING INCLINATION OF EARTH SATELLITES USING THE GRAVITY OF THE MOON
}

\author{
KARLA DE SOUZA TORRES AND A. F. B. A. PRADO
}

Received 23 August 2005; Revised 14 April 2006; Accepted 18 April 2006

We analyze the problem of the orbital control of an Earth's satellite using the gravity of the Moon. The main objective is to study a technique to decrease the fuel consumption of a plane change maneuver to be performed in a satellite that is in orbit around the Earth. The main idea of this approach is to send the satellite to the Moon using a singleimpulsive maneuver, use the gravity field of the Moon to make the desired plane change of the trajectory, and then return the satellite to its nominal semimajor axis and eccentricity using a bi-impulsive Hohmann-type maneuver. The satellite is assumed to start in a Keplerian orbit in the plane of the lunar orbit around the Earth and the goal is to put it in a similar orbit that differs from the initial orbit only by the inclination. A description of the close-approach maneuver is made in the three-dimensional space. Analytical equations based on the patched conics approach are used to calculate the variation in velocity, angular momentum, energy, and inclination of the satellite. Then, several simulations are made to evaluate the savings involved. The time required by those transfers is also calculated and shown.

Copyright (C 2006 K. de Souza Torres and A. F. B. A. Prado. This is an open access article distributed under the Creative Commons Attribution License, which permits unrestricted use, distribution, and reproduction in any medium, provided the original work is properly cited.

\section{Introduction}

On Christmas Day, 1997, the AsiaSat 3 satellite was launched from the Baikonur Cosmodrome in Kazakhstan to provide communications and television services for an Asian company. The launch of the satellite malfunctioned, however, leaving the satellite in a useless, highly inclined elliptical orbit, without enough fuel on the satellite itself to reach the proper orbit. Although the satellite itself was functional, insurers declared it a total loss for its original purposes. The ownership of the satellite was transferred to Hughes Global Services Inc. to try a very special maneuver. Hughes orbital engineers devised a novel mission to save the satellite, using lunar gravity. The experiment was the first known lunar mission involving a communications satellite (http://www.g2satellitesolutions.com/). 
The goal of the majority of the space programs in the world is to develop techniques to decrease the fuel expenditure in space missions. Sometimes, a mission must be projected in an emergency case to rescue a satellite, as the AsiaSat 3 satellite. In that case, the fuel was not enough to transfer the satellite to its proper orbit. However, although there was not sufficient combustible to change the orbital inclination of the satellite (an expensive maneuver), it was enough to send the satellite to the Moon, and to leave the gravity field make the desired plane change, without fuel consumption.

In this paper we analyze the problem of the orbital control of a terrestrial satellite using the lunar gravity. Analytical equations are developed to quantify the variations of inclination, velocity, energy, and angular momentum of a close approximation with the Moon, and several simulations are made to evaluate the savings involved. The main idea is to verify in which cases this strategy is more economical than classic methods (that do not use the swing-by).

The maneuver consists in three parts: (i) application of a first impulse that is used to send the satellite in a planar trajectory to the Moon; (ii) the swing-by with the Moon is used to make a free plane change; (iii) two impulses are applied to return the satellite to the desired nominal values of semimajor axes and eccentricity. The main advantage of this maneuver is that the change of orbital plane is achieved through the lunar gravity, saving a great amount of fuel and performing missions that otherwise were not practicable. Another advantage of this approach is that it allows the possibility of studying the Moon, making the mission a double-objective one.

\section{The proposed maneuver}

It is assumed that the system has three bodies: a primary $\left(M_{1}\right.$, the Earth) and a secondary $\left(M_{2}\right.$, the Moon) bodies with finite masses and a third body with negligible mass (the satellite) that has its motion governed by the two other bodies. The problem is divided in three phases dominated by the two-body celestial mechanics ("patched-conics" approach). It is assumed that the satellite is in an initial orbit around the Earth that is coplanar with the orbit of the Moon, with given semimajor axis $\left(a_{0}\right)$ and eccentricity $\left(e_{0}\right)$ such that this orbit is coplanar with the orbit of the Moon.

The canonical system of units is used, implying that: (i) the unit of distance is the Earth-Moon distance $(384400.05 \mathrm{~km})$; (ii) the angular velocity of the motion of the Earth and the Moon is assumed to be equal to one; (iii) the mass of the Moon is given by $\mu_{L}=m_{2} /\left(m_{1}+m_{2}\right)$ (where $m_{1}$ and $m_{2}$ are the real masses of the Earth and the Moon, resp.) and the mass of the Earth $\left(\mu_{T}\right)$ is $\left(1-\mu_{L}\right)$, being the total mass of the system equal to one; (iv) the unit of time is defined such that the period of the motion of the Earth and the Moon is $2 \pi ;(\mathrm{v})$ the gravitational constant is equal to one.

2.1. The initial impulse. The first step is to apply a first impulse $\left(\Delta V_{1}\right)$ to send the satellite from its initial parking orbit around the Earth to the Moon. The minimum value for the semimajor axis $\left(a_{1}\right)$ that allows a transfer to the Moon is given by $a_{1}=\left(1+a_{0}\right) / 2$. As the initial orbit around the Earth is elliptical, a point in the orbit where the impulse will be applied must be chosen. Due to change of energy reasons, the most economical point to apply the impulse is the perigee of the orbit. In this way, the magnitude of the impulse 


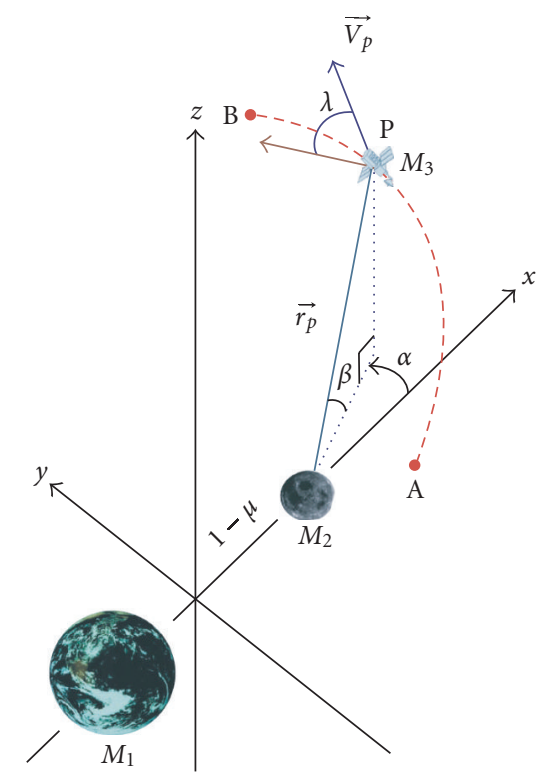

Figure 2.1. The swing-by in three dimensions.

required for the transfer and the eccentricity of the transfer orbit are

$$
\begin{gathered}
\Delta V_{1}=\sqrt{\frac{2 \mu_{T}}{a_{0}\left(1-e_{0}\right)}-\frac{\mu_{T}}{a_{1}}}-\sqrt{\frac{2 \mu_{T}}{a_{0}\left(1-e_{0}\right)}-\frac{\mu_{T}}{a_{0}}}, \\
e_{1}=1-\frac{a_{0}\left(1-e_{0}\right)}{a_{1}} .
\end{gathered}
$$

2.2. The swing-by maneuver. The second step is to quantify the maneuver when the satellite approaches the Moon (the swing-by). The motion of the satellite is then governed by the secondary body $M_{2}$, since the system follows the two-body model. The radius of the sphere of influence of the Moon is obtained from the equations shown in reference [3]. Figure 2.1 shows the sequence for this maneuver.

The variables used to identify uniquely the swing-by trajectory are $r_{p}$, the distance from the satellite to the center of the Moon at the moment of the closest approximation to this body (perilune distance); $V_{p}$, the velocity of the satellite at perilune; two angles ( $\alpha$ and $\beta$ ) that specify the direction of the perilune in a three-dimensional space; $\lambda$, the angle between the velocity vector $\vec{V}_{p}$ and the horizontal plane that passes by the perilune. The satellite leaves point $\mathrm{A}$, passes by point $\mathrm{P}$ (the perilune), and goes to point $\mathrm{B}$. Points $\mathrm{A}$ and $B$ are chosen in a such way that the influence of the Moon at those two points can be neglected and, consequently, the energy can be assumed to remain constant after B and before A. The result of this maneuver is a change in velocity, energy, angular momentum, 


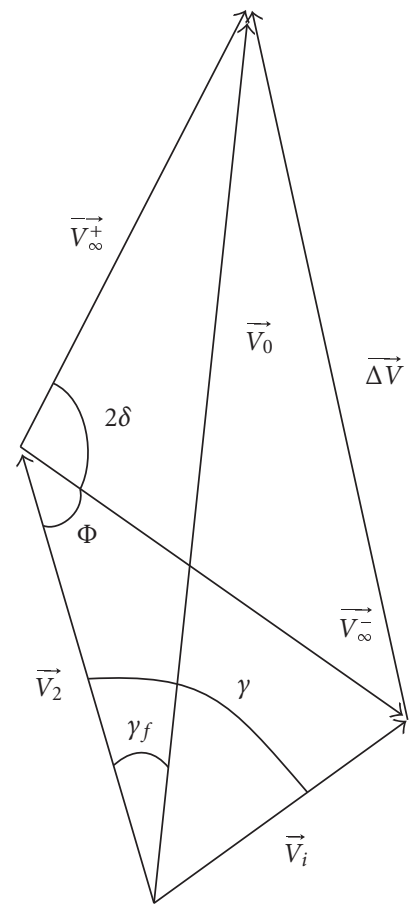

Figure 2.2. Vector addition during the swing-by.

and inclination of the Keplerian orbit of the satellite around the Earth. Figure 2.2 shows the vector addition of the velocities involved in the swing-by.

In Figure 2.2, $\vec{V}_{i}$ and $\vec{V}_{O}$ are the velocity vectors of the satellite with respect to the Earth (inertial velocities) immediately before and after the close approximation, respectively; $\gamma$ is the flight path angle, that is, the angle between the inertial velocity of the particle and the velocity of the Moon; $\delta$ is half of the turning angle of the particle around the Moon; $\vec{V}_{\infty}$ is the velocity vector of the particle with respect to the Moon at the moment when the approach starts $(-)$ or ends $(+)\left(\vec{V}_{\infty}^{-}\right.$and $\vec{V}_{\infty}^{+}$have the same magnitude); and $\Delta \vec{V}$ is the total variation in the velocity of the satellite caused by the swing-by maneuver. Note that $\Delta \vec{V}=\vec{V}_{o}-\vec{V}_{i}=\left(\vec{V}_{\infty}^{+}+\vec{V}_{2}\right)-\left(\vec{V}_{\infty}^{-}+\vec{V}_{2}\right)$.

Then it is possible to calculate the magnitude of $\vec{V}_{i}$, as well as the true anomaly of that point:

$$
\begin{gathered}
V_{i}=\sqrt{\mu_{T}\left(2-\frac{1}{a_{1}}\right)}, \\
\theta=\cos ^{-1}\left[\frac{1}{e_{1}}\left(a_{1}\left(1-e_{1}^{2}\right)-1\right)\right] .
\end{gathered}
$$


In these equations, only the positive value of the true anomaly was taken, and we are using the fact that the Earth-Moon distance is equal to one. Next, the flight path angle $\gamma$ as well as the magnitude of $\vec{V}_{\infty}$ is calculated. Assuming a counterclockwise orbit for the particle, these parameters can be written as follows:

$$
\begin{gathered}
\gamma=\tan ^{-1}\left[\frac{e_{1} \sin \theta}{1+e_{1} \cos \theta}\right], \\
V_{\infty}=\sqrt{V_{i}^{2}+V_{2}^{2}-2 V_{i} V_{2} \cos \gamma} .
\end{gathered}
$$

The angle $\phi$ shown in Figure 2.2 and the turning angle $(2 \delta)$ of the particle around the Moon are

$$
\begin{gathered}
\phi=\cos ^{-1}\left[-\frac{V_{i}^{2}-V_{2}^{2}-V_{\infty}^{2}}{2 V_{2} V_{\infty}}\right], \\
\delta=\sin ^{-1}\left(1 / \frac{1+r_{p} V_{\infty}^{2}}{\mu_{L}}\right) .
\end{gathered}
$$

The angle of approach $(\alpha)$ (see Figure 2.1) is given by $\alpha=\pi+\phi+\delta$. Assuming that the satellite trajectory is in the same plane of the orbit of the Moon around the Earth and that the angle $\beta$ and the perilune distance $r_{p}$ are used as control variables of the maneuver (they can be reached with maneuvers that have negligible consumption), we can obtain the following relation for the angle $\lambda$ of Figure 2.1:

$$
\lambda=\sin ^{-1}[-\operatorname{tg}(\delta) \operatorname{tg}(\beta)]
$$

The equations for the inertial velocities $\vec{V}_{i}$ and $\vec{V}_{o}$ are [2]

$$
\begin{aligned}
\vec{V}_{i}=\vec{V}_{\infty}^{-}+\vec{V}_{2}= & V_{\infty} \sin \delta(\cos \beta \cos \alpha, \cos \beta \sin \alpha, \sin \beta) \\
& +V_{\infty} \cos \delta(-\sin \lambda \sin \beta \cos \alpha-\cos \lambda \sin \alpha-\sin \lambda \sin \beta \sin \alpha \\
& +\cos \lambda \cos \alpha, \cos \beta \sin \lambda)+\left(0, V_{2}, 0\right), \\
\vec{V}_{o}=\vec{V}_{\infty}^{+}+\vec{V}_{2}= & -V_{\infty} \sin \delta(\cos \beta \cos \alpha, \cos \beta \sin \alpha, \sin \beta) \\
& +V_{\infty} \cos \delta(-\sin \lambda \sin \beta \cos \alpha-\cos \lambda \sin \alpha,-\sin \lambda \sin \beta \sin \alpha \\
& +\cos \lambda \cos \alpha, \cos \beta \sin \lambda)+\left(0, V_{2}, 0\right) .
\end{aligned}
$$


6 Changing inclination using the gravity of the moon

From the above equations, it is possible to obtain expressions for the variations in velocity, energy, and angular momentum of the satellite. They are

$$
\begin{gathered}
\Delta V=|\Delta \vec{V}|=\left|\vec{V}_{0}-\vec{V}_{i}\right|=\left|-2 V_{\infty} \sin \delta(\cos \alpha \cos \beta, \cos \beta \sin \alpha, \sin \beta)\right|=2 V_{\infty} \sin \delta \\
\Delta E=\frac{1}{2}\left(V_{o}^{2}-V_{i}^{2}\right)=-2 V_{2} V_{\infty} \cos \beta \sin \alpha \sin \delta \\
\vec{C}_{i}=\vec{R} \times \vec{V}_{i}=d V_{\infty}\left(0,-\sin \beta \sin \delta+\cos \beta \cos \delta \sin \lambda, \frac{V_{2}}{V_{\infty}}\right. \\
+\cos \alpha \cos \delta \cos \lambda+\cos \beta \sin \alpha \sin \delta-\cos \delta \sin \alpha \sin \beta \sin \lambda) \\
\vec{C}_{o}=\vec{R} \times \vec{V}_{o}=d V_{\infty}\left(0, \sin \beta \sin \delta-\cos \beta \cos \delta \sin \lambda, \frac{V_{2}}{V_{\infty}}+\cos \alpha \cos \delta \cos \lambda\right. \\
-\cos \beta \sin \alpha \sin \delta-\cos \delta \sin \alpha \sin \beta \sin \lambda) \\
\begin{array}{c}
\Delta C=\left|\vec{C}_{o}-\vec{C}_{i}\right|=\left|2 d V_{\infty} \sin \delta(0, \sin \beta,-\cos \beta \sin \alpha)\right| \\
=2 d V_{\infty} \sin \delta\left(\cos { }^{2} \beta \sin { }^{2} \alpha+\sin { }^{2} \beta\right)^{1 / 2}
\end{array}
\end{gathered}
$$

In these equations, $\vec{R}=(d, 0,0)$ is the position vector of $M_{2}$, and $d$ is the Earth-Moon distance. The inclination before the close approach is assumed to be zero and the inclination after the passage is

$$
\begin{aligned}
\operatorname{Cos}\left(i_{o}\right) & =\frac{C_{o Z}}{\left|\vec{C}_{o}\right|} \\
& =\left(1+\left(\frac{\sin \beta \sin \delta-\cos \beta \cos \delta \sin \lambda}{V_{2} / V_{\infty}+\cos \alpha \cos \delta \cos \lambda-\cos \beta \sin \alpha \sin \delta-\cos \delta \sin \alpha \sin \beta \sin \lambda}\right)^{2}\right)^{-1 / 2},
\end{aligned}
$$

where $\vec{C}_{o}$ is the final angular momentum (subscript $Z$ stands for the $z$-component) and $i_{o}$ is the final inclination.

2.3. The final impulses. After the swing-by, two impulses are applied to return the satellite to the desired nominal values of semimajor axes and eccentricity. In other words, it must return the satellite to the initial orbital size and shape, $a_{0}$ and $e_{0}$. 
The semimajor axis $\left(a_{2}\right)$ and the eccentricity $\left(e_{2}\right)$ of the orbit after the close approach are given by

$$
\begin{aligned}
& a_{2}=\frac{\mu_{T}}{2 \mu_{T}-V_{o}^{2}}, \\
& e_{2}=\sqrt{1-\frac{C_{o}^{2}}{\mu_{T} a_{2}}} .
\end{aligned}
$$

The semimajor axis $\left(a_{3}\right)$ of the orbit after the second impulse is now calculated. It is

$$
a_{3}=\frac{a_{2}\left(1+e_{2}\right)+a_{0}\left(1-e_{0}\right)}{2} .
$$

Then, the second and third impulses that will put the satellite in the final desired orbit are

$$
\begin{gathered}
\Delta V_{2}=\sqrt{\frac{2 \mu_{T}}{a_{2}\left(1+e_{2}\right)}-\frac{\mu_{T}}{a_{2}}}-\sqrt{\frac{2 \mu_{T}}{a_{2}\left(1+e_{2}\right)}-\frac{\mu_{T}}{a_{3}}}, \\
\Delta V_{3}=\sqrt{\frac{2 \mu_{T}}{a_{0}\left(1-e_{0}\right)}-\frac{\mu_{T}}{a_{3}}}-\sqrt{\frac{2 \mu_{T}}{a_{0}\left(1-e_{0}\right)}-\frac{\mu_{T}}{a_{0}}} .
\end{gathered}
$$

So, the maneuver is accomplished and the satellite will be in a final orbit with the same semimajor axis and eccentricity of the initial one, but with an inclination $i_{o}$ with respect to the orbital plane of the Moon. To obtain the total cost of the proposed maneuver the three impulses must be added:

$$
\Delta V_{T}=\Delta V_{1}+\Delta V_{2}+\Delta V_{3}
$$

\section{Classical maneuvers}

A plane change maneuver rotates the orbital plane in inertial space while holding its size and shape, $a$ and $e$. We are now going to compare the orbital maneuver exposed above with the classical plane change maneuvers: the single and the three-impulse maneuvers [1].

The single-impulse plane change maneuver is characterized by the application of one impulse in the satellite when it passes by the intersection of the two orbits. The best case for this maneuver is when the impulse is given at the apogee of the orbit. With it, the impulse required is

$$
\Delta V_{\text {Mono }}=2 \sqrt{\frac{2 \mu_{T}}{a_{0}\left(1+e_{0}\right)}-\frac{\mu_{T}}{a_{0}}} \sin \frac{i}{2} .
$$

In the three-impulse plane change maneuver the first impulse $\Delta V_{\text {Tri } 1}$ is added tangentially to the orbital velocity in order to achieve a transfer ellipse whose apogee radius is $r_{2}$ ( $r_{1}$ is the perigee). At the apogee, $\Delta V_{\text {Tri2 }}$ is used to rotate the apogee velocity through the 
desired plane change angle $i$. The equations for this maneuver are

$$
\begin{gathered}
\Delta V_{\operatorname{Tri} 1}=\Delta V_{\operatorname{Tri} 3}=\sqrt{\frac{2 \mu_{T}}{r_{1}}-\frac{2 \mu_{T}}{\left(r_{1}+r_{2}\right)}}-\sqrt{\frac{2 \mu_{T}}{r_{1}}-\frac{\mu_{T}}{a_{0}}}, \\
\Delta V_{\operatorname{Tri} 2}=2 \sqrt{\frac{2 \mu_{T}}{r_{2}}-\frac{2 \mu_{T}}{\left(r_{1}+r_{2}\right)}} \sin \frac{i}{2},
\end{gathered}
$$

where $r_{1}=a_{0}\left(1-e_{0}\right)$, and the total cost is given by

$$
\Delta V_{\operatorname{Tri}}=\Delta V_{\operatorname{Tr} 11}+\Delta V_{\operatorname{Tr} 2}+\Delta V_{\operatorname{Tri} 3}
$$

\section{Results}

To obtain the results, the total cost of the proposed maneuver $\Delta V_{T}$ (2.7) was compared with that of the classical maneuvers, varying the values of the free parameters: $e_{0}, a_{0}, r_{p}$, and the $\beta$ angle. The effect of the variation of the parameter $a_{1}$ can also be tested, but in this paper just the minimum value is used. So, several simulations were performed with the equations shown above.

Figure 4.1 shows the inclination change as a function of the angle $\beta$, in radians, obtained after the swing-by maneuver for five values of eccentricity $e_{0}$. It is important to note that the inclination change is proportional to this variable. In Figures 4.1-4.4 there are some discontinuities. Physically, those points split the plots in two regions: the one where the values of the angle $\beta$ can be achieved with the given initial conditions and the one where those values cannot be achieved with the given initial conditions.

The inclinations obtained after the swing-by are less than 1 radian for values of the eccentricity $e_{0}$ less than 0.5 , that is, just for larger values of initial eccentricity it gets inclinations change larger than 1 radian. So, the three-impulse plane change maneuver will not be used in the comparisons with the proposed maneuver, since this maneuver is worse than the monoimpulse change maneuver for the values of the inclination change that are obtained here.

Figure 4.2 shows the difference in fuel consumption comparing the proposed maneuver with the single-impulse maneuver $\left(\Delta V_{t}-\Delta V_{\text {mono }}\right)$ measured in canonical units of increment of velocity to be applied to the satellite ( 1 canonical unit is equal to $1.023 \mathrm{~km} / \mathrm{s}$ ) as a function of the angle $\beta$, in radians, for the same five values of eccentricity of Figure 4.1. It is visible that the proposed maneuver is better when the initial orbits are next to the circular ones.

Next, the effect of the perilune distance $r_{p}$ is analyzed. In this case, one value of eccentricity is chosen $\left(e_{0}=0.01\right)$ and five values of $r_{p}$ are shown in Figure 4.3. The results show that two regions can be distinguished. In the region $\beta \sim \pi$, the advantages of the swing-by maneuver increase when this parameter decreases. But in the region $\beta \sim 1$ (or $\beta \sim 5$ ), the best value of $r_{p}$ is 0.04566 that represents a saving of 0.467501 canonical units of the proposed maneuver over the classical one.

Then, the effects of varying the initial semimajor axis $a_{0}$ are considered. In that case, the semimajor axis $a_{1}$ is the minimum possible in each case. Figure 4.4 shows the same differences in fuel consumption as a function of $\beta$ considering five values for $a_{0}$ with the 


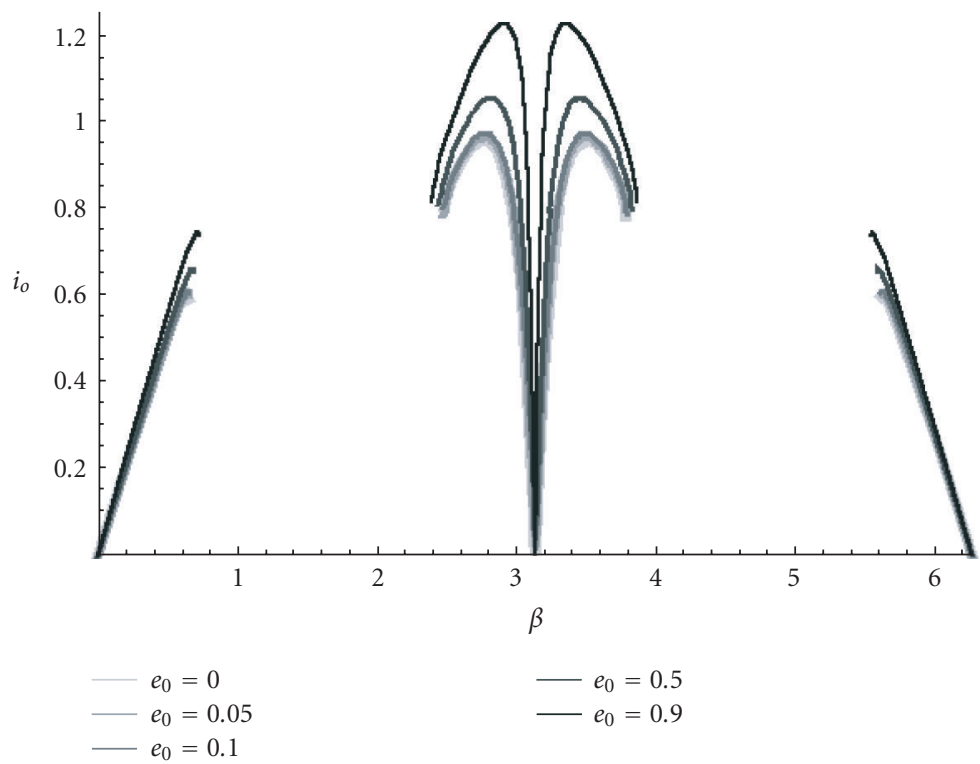

Figure 4.1. Inclination change as a function of $\beta$ for five values of $e_{0}\left(a_{0}=0.017, a_{1}=0.51\right.$, and $\left.r_{p}=0.0046\right)$.

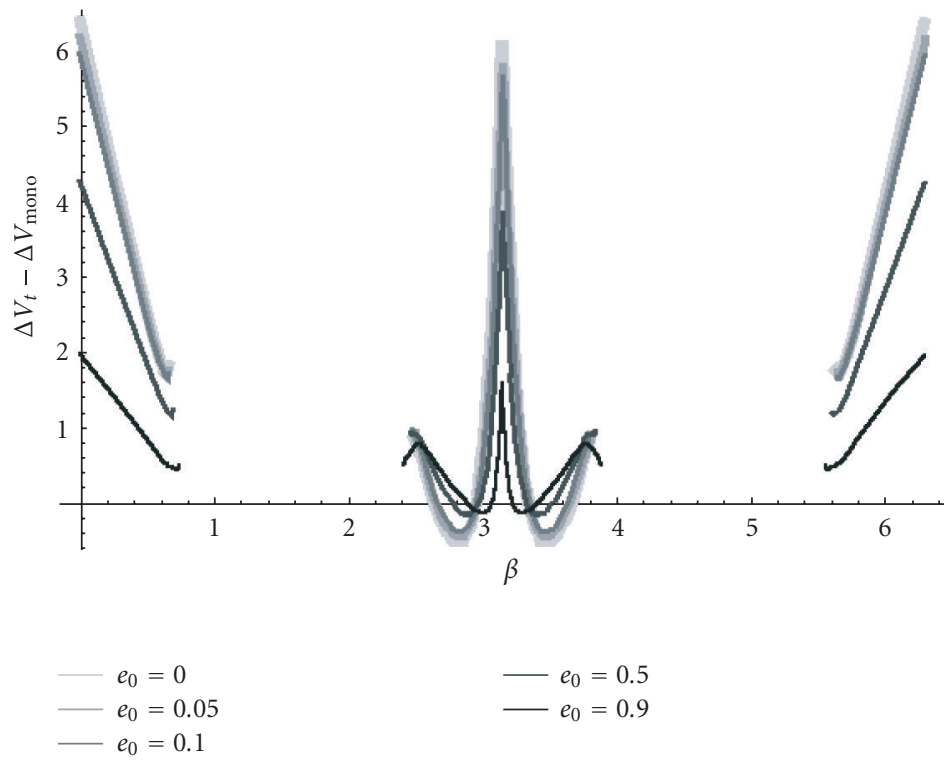

Figure 4.2. ( $\left.\Delta V_{t}-\Delta V_{\text {mono }}\right)$ as a function of $\beta$ for five values of $e_{0}\left(a_{0}=0.017, a_{1}=0.51\right.$, and $r_{p}=$ $0.0046)$. 


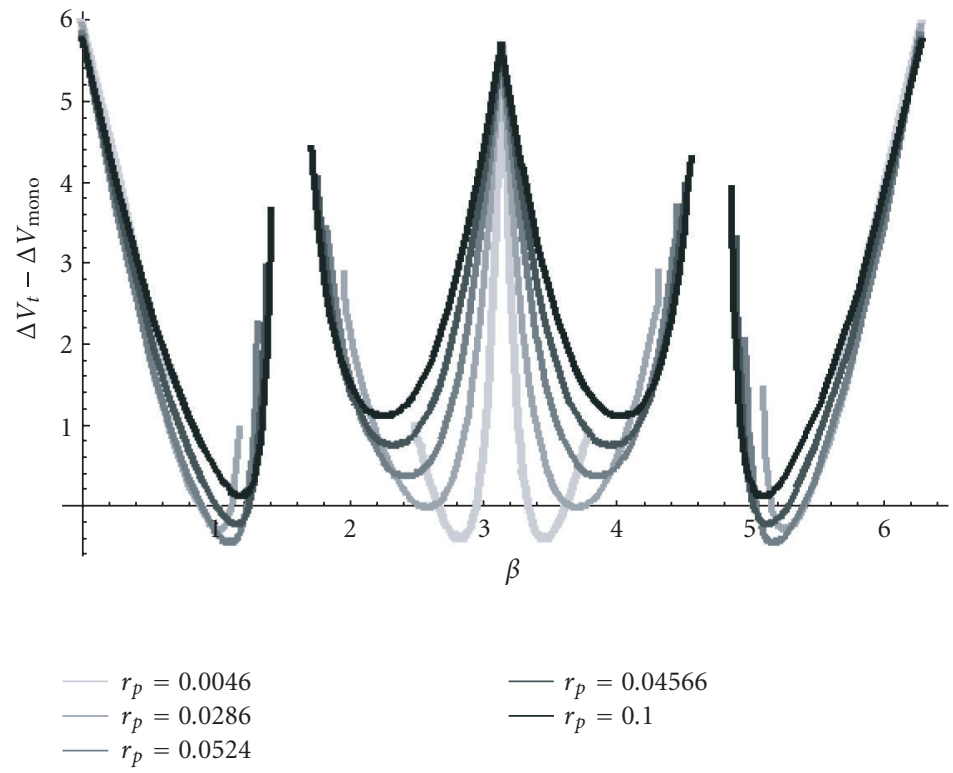

Figure 4.3. $\left(\Delta V_{t}-\Delta V_{\text {mono }}\right)$ as a function of $\beta$ for five values of $r_{p}\left(e_{0}=0.01, a_{0}=0.017\right.$, and $a_{1}=$ $0.51)$.

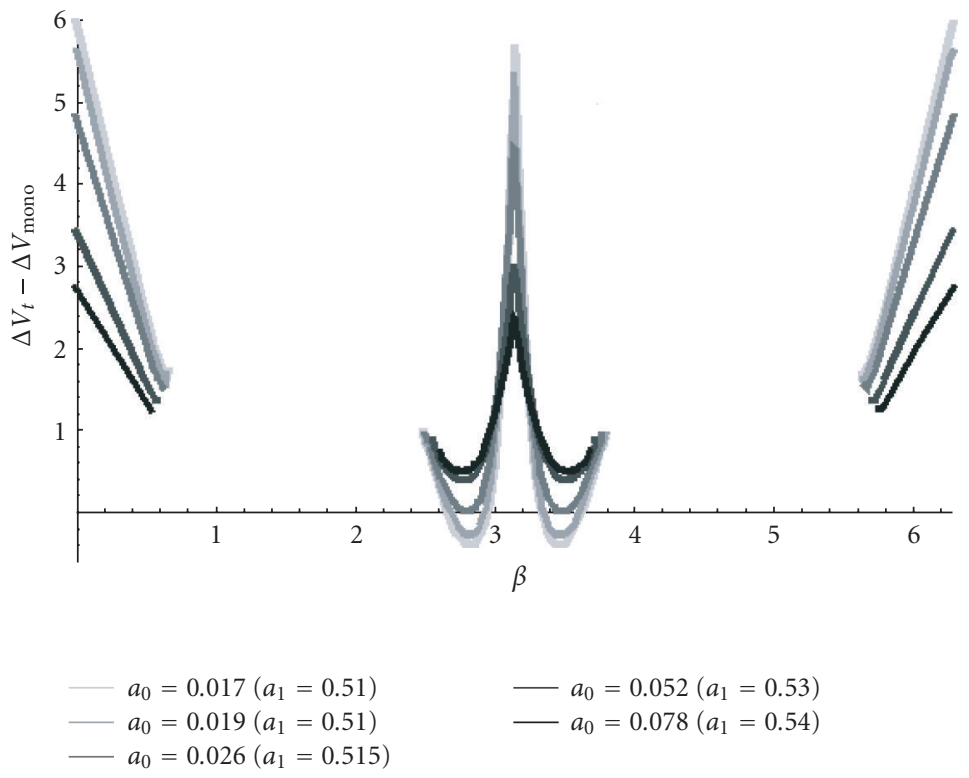

Figure 4.4. $\left(\Delta V_{t}-\Delta V_{\text {mono }}\right)$ as a function of $\beta$ for five values of $a_{0}$ and minimum values of $a_{1}\left(e_{0}=\right.$ 0.01 , and $\left.r_{p}=0.0046\right)$. 


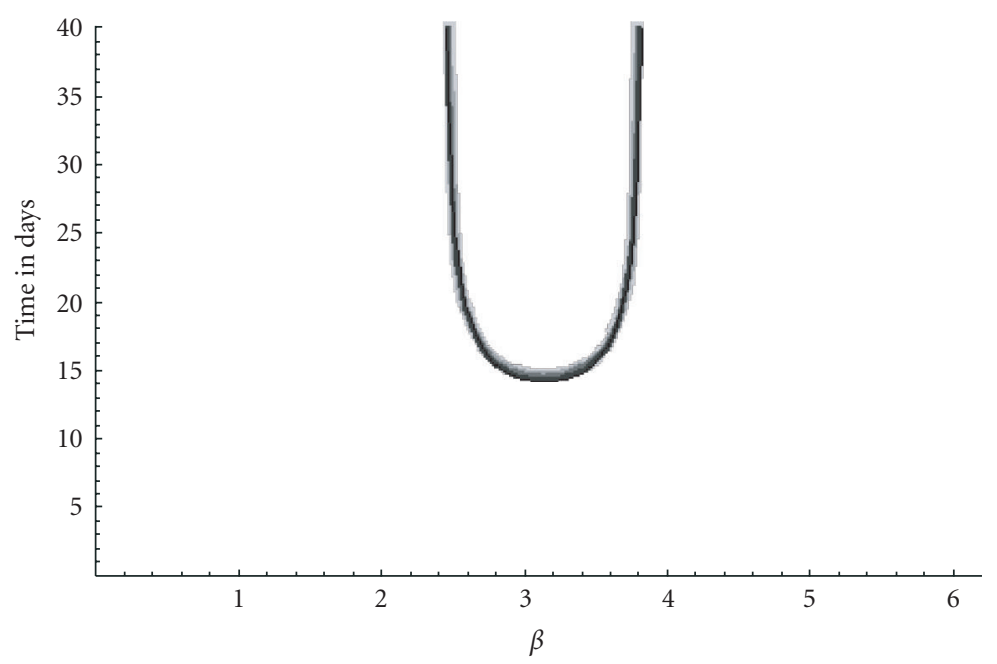

Figure 4.5. Total time in days as a function of $\beta$ for five values of $e_{0}\left(a_{0}=0.017, a_{1}=0.51\right.$, and $r_{p}=$ $0.0046)$.

respective possible minimum $a_{1}$. It is clear that the savings of the swing-by maneuver decrease when the value of this variable increases. This result is expected because the costs of the inclination change increase very much when the initial orbit gets closer to the Earth.

In the next figures, the total time to perform the proposed maneuver is shown. Figure 4.5 shows the time, in days, as a function of the angle $\beta$, in radians, for the same five values of eccentricity used to build Figure 4.2. The total time is independent of the initial orbit eccentricity. In the region $\beta \sim 1$ (or $\beta \sim 5$ ), the proposed maneuver has values very large for the total time, so it is not shown in the figure. Figure 4.6 considers the effect of the perilune distance $r_{p}$. Finally, Figure 4.7 shows the effects of varying the initial semimajor axis $a_{0}$ in the total time for the transfer.

\section{Conclusions}

An alternative maneuver is presented to perform a plane change maneuver using the lunar gravity to save fuel. Elliptic initial orbits are considered and analyzed. A set of analytical equations is used to describe the maneuver and with them it is possible to compare it with the classical plane change maneuvers. The results show that in several circumstances the proposed maneuver can be more economical than the standard maneuvers if a proper choice of values for the free parameters is made: (i) the orbit is next to circular, (ii) the semimajor axis of the initial orbit has the smallest possible values, (iii) the perilune distance of the close approach is as small as possible if the angle $\beta \sim \pi$ and it is equal to 0.04566 ( $\sim 15813 \mathrm{~km}$ above lunar surface) if $\beta \sim 1$ or $\beta \sim 5$. This research provides a theoretical base for space missions that includes change plane maneuver like the AsiaSat 3 rescue. To be used in real missions, perturbations effects must be included and 
12 Changing inclination using the gravity of the moon

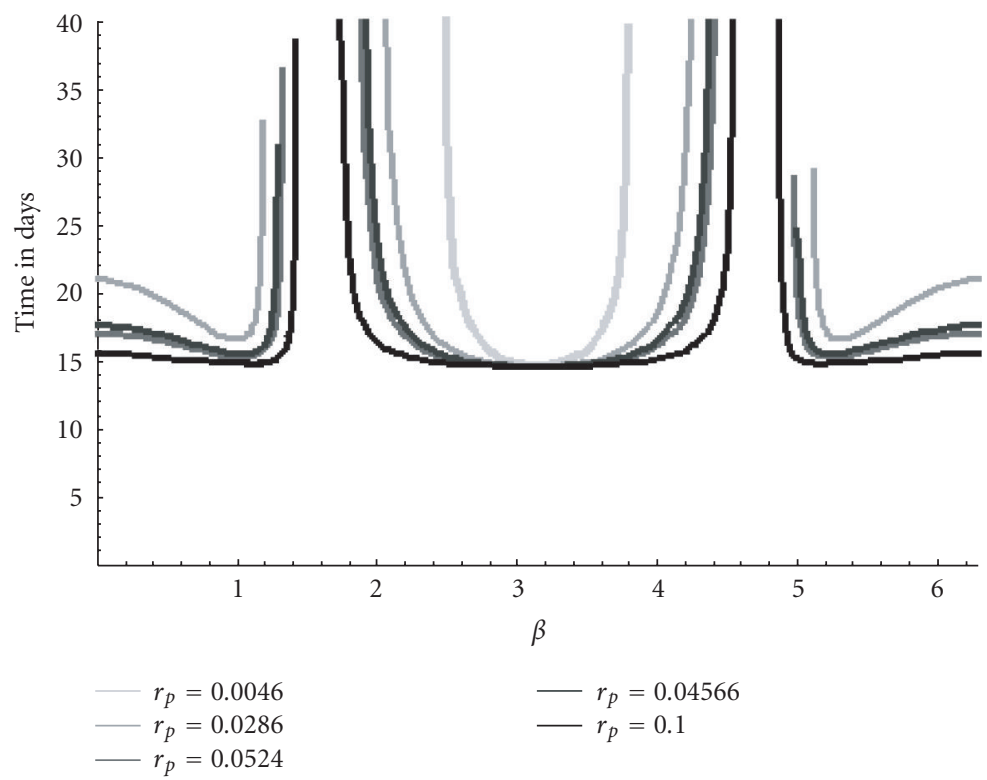

Figure 4.6. Total time in days as a function of $\beta$ for five values of $r_{p}\left(e_{0}=0.01, a_{0}=0.017\right.$, and $a_{1}=$ $0.51)$.

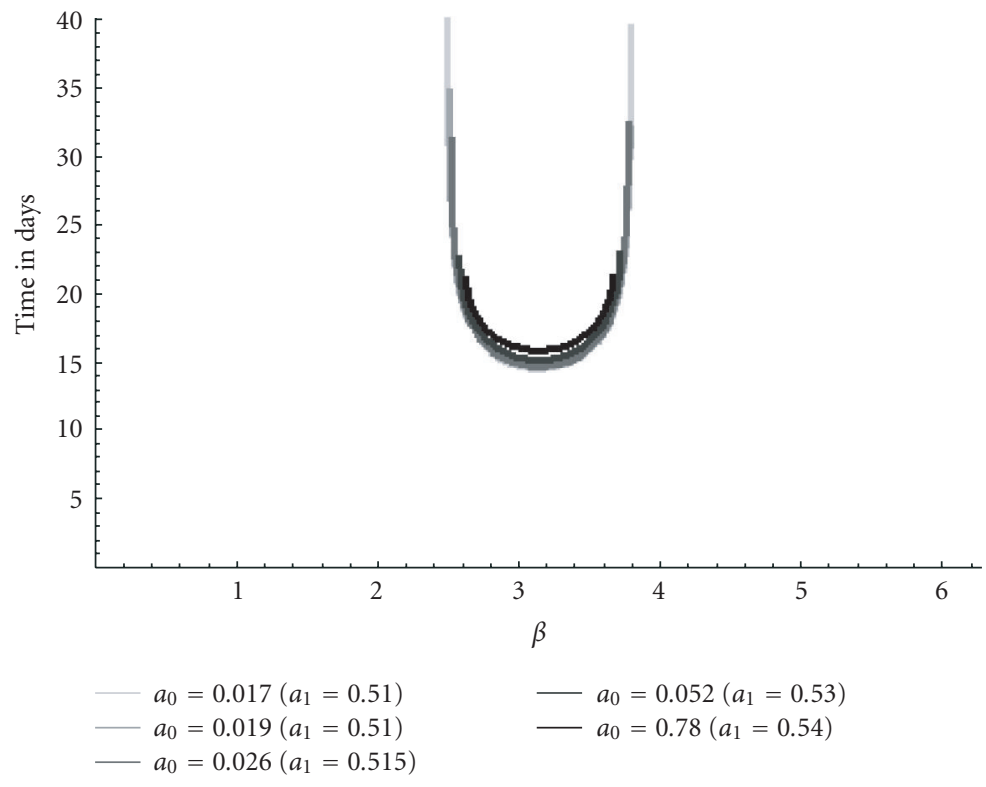

Figure 4.7. Total time in days as a function of $\beta$ for five values of $a_{0}$ and minimum values of $a_{1}$ $\left(e_{0}=0.01\right.$, and $\left.r_{p}=0.0046\right)$. 
a suggestion for future researches is to improve this model adding such effects to make it practical.

\section{Acknowledgment}

The authors are grateful to the FAPESP (Foundation to Support Research in the São Paulo State) for supporting this research under contracts 01/10193-3 and 03/03262-4.

\section{References}

[1] V. A. Chobotov, Orbital Motion, 2nd ed., American Institute of Aeronautics and Astronautics, Virginia, 1996.

[2] A. F. B. A. Prado, An analytical description of the close approach maneuver in three dimensions, Proceedings of the 51st International Astronautical Congress (IAF-00-A.5.05), Rio de Janeiro, October 2000.

[3] A. E. Roy, Orbital Motion, 3rd ed., Institute of Physics, Bristol, 1988.

Karla de Souza Torres: Divisão de Mecânica, Instituto Nacional de Pesquisas Espaciais, Avenida dos Astronautas 1758, São José dos Campos, São Paulo, 12227-010, Brazil E-mail addresses: karla.souza@dem.inpe.br; karlchen79@gmail.com

A. F. B. A. Prado: Divisão de Mecânica, Instituto Nacional de Pesquisas Espaciais, Avenida dos Astronautas 1758, São José dos Campos, São Paulo, 12227-010, Brazil E-mail address: prado@dem.inpe.pr 


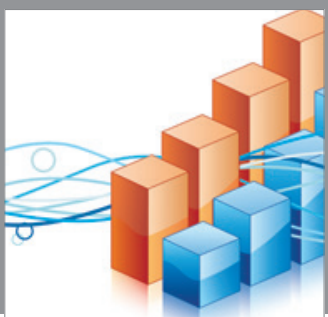

Advances in

Operations Research

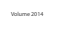

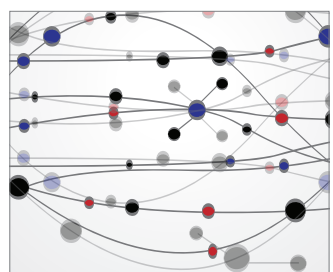

\section{The Scientific} World Journal
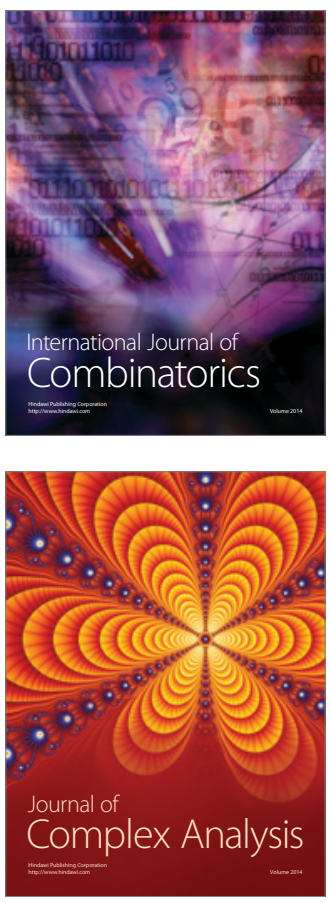

International Journal of

Mathematics and

Mathematical

Sciences
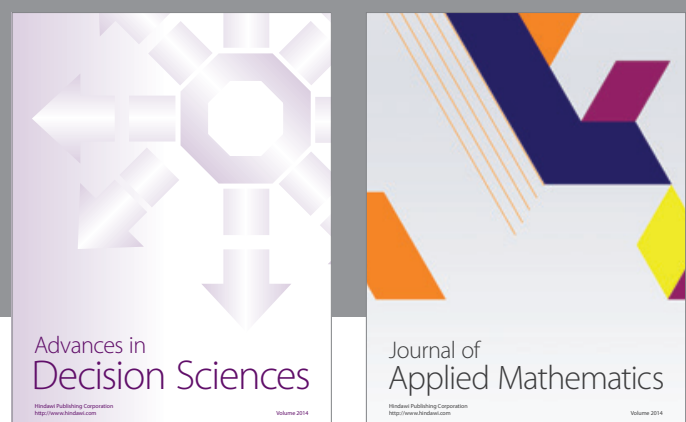

Journal of

Applied Mathematics
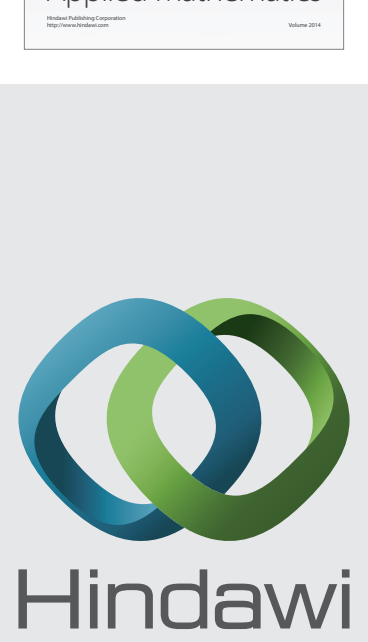

Submit your manuscripts at http://www.hindawi.com
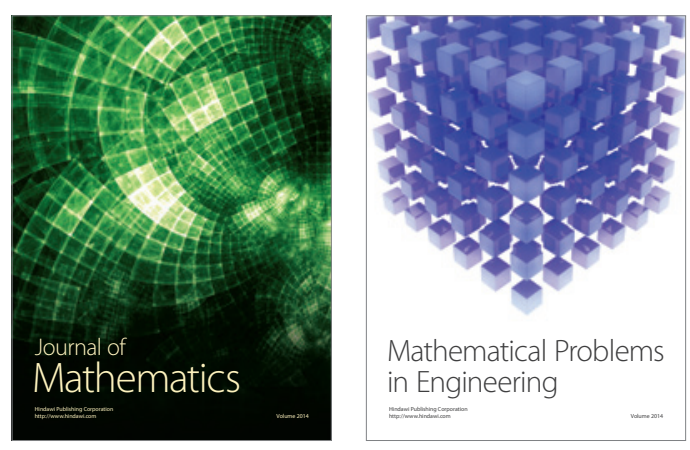

Mathematical Problems in Engineering
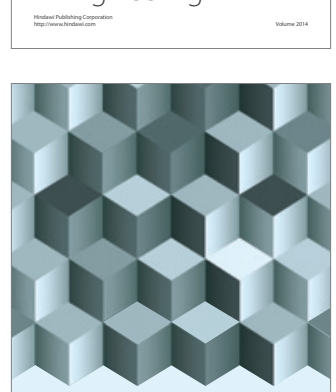

Journal of

Function Spaces
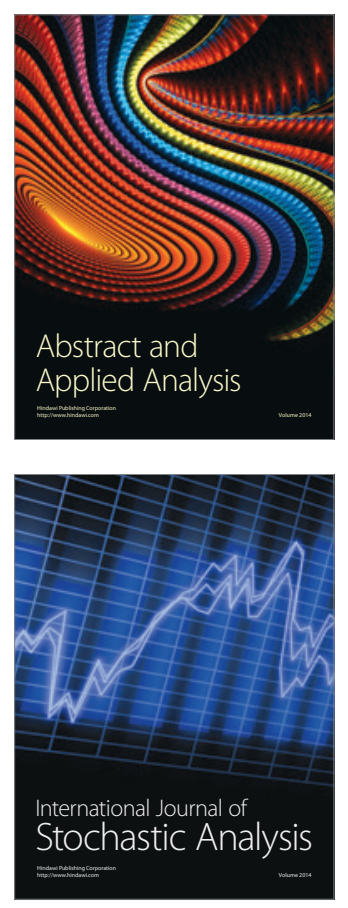

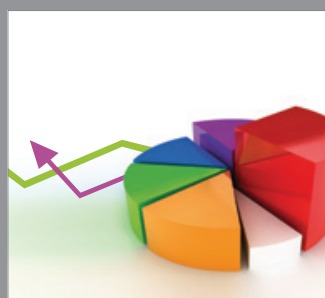

ournal of

Probability and Statistics

Promensencen
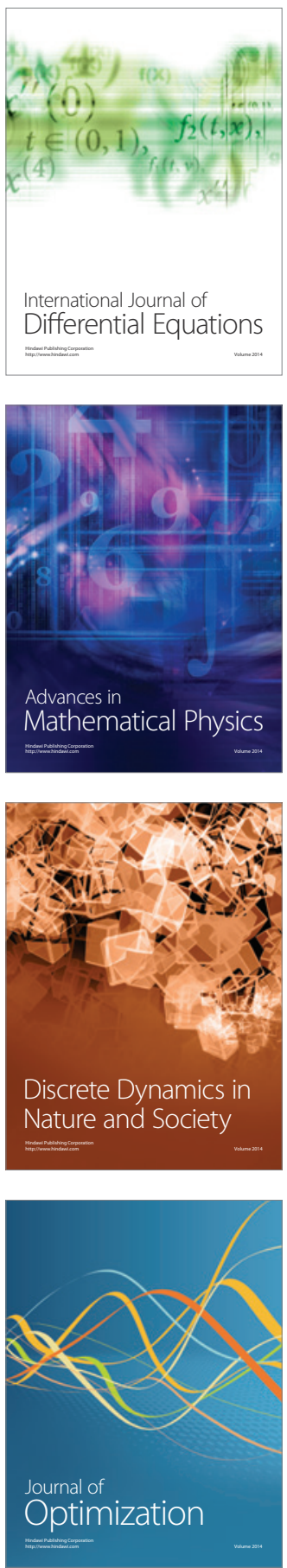